\title{
Vacancy Engineering to Regulate Photocatalytic Activity of Polymer Photosensitizers for Amplifying Photodynamic Therapy against Hypoxic Tumors
}

Jing Bai, ${ }^{a}$ Chengjia Peng, ${ }^{a}$ Wenjia Lv, ${ }^{a}$ Jingju Liu, ${ }^{*}$ a Yashuang Hei ${ }^{*}$ a and Xiangjie $B o * a$

a Key Laboratory of Polyoxometalate and Reticular Material Chemistry of Ministry of Education, National \& Local United Engineering Laboratory for Power Batteries, Key Laboratory of Nanobiosensing and Nanobioanalysis at Universities of Jilin Province, Analysis and Testing Center, Department of Chemistry, Northeast Normal University, Changchun, Jilin Province 130024, China

Corresponding author.

*E-mail:

liujj132@,nenu.edu.cn (J.L.);

heiys505@nenu.edu.cn (Y.H.);

baoxj133@nenu.edu.cn (M.Z.). 
Chemicals and reagents. Thionine (Th), iron (III) chloride hexahydrate $\left(\mathrm{FeCl}_{3} \cdot 6 \mathrm{H}_{2} \mathrm{O}\right)$, methyl red (MR), $\left[\mathrm{Ru}(\mathrm{dpp})_{3}\right] \mathrm{Cl}_{2}$ (RDPP), gold (III) chloride trihydrate $\left(\mathrm{HAuCl}_{4} \cdot 3 \mathrm{H}_{2} \mathrm{O}\right)$, and hydrogen peroxide $\left(\mathrm{H}_{2} \mathrm{O}_{2}, 30 \%\right)$ were purchased from Aladdin Reagent Co., Ltd. (Shanghai, China). 3-(4, 5-Dimethylthiazol-2-yl)-2, 5-diphenyltetrazolium bromide (MTT), fetal bovine serum (FBS), Dulbecco's modified Eagle's medium (DMEM), penicillin-streptomycin $(100 \times)$ and dimethyl sulfoxide (DMSO, 99\%) were obtained from Dingguo Reagent Co., Ltd. (Changchun, China). 5,5Dimethyl-1-pyrroline N-oxide (DMPO), terephthalic acid (TA), filipin, amiloride and 2, 2, 6, 6-tetramethylpiperidine (TEMP) were purchased from Macklin Biochemical Co., Ltd. (Shanghai, China). Singlet oxygen sensor green (SOSG), calcein acetoxymethyl ester (calcein AM), hydroxyphenyl fluorescein (HPF), 2', 7'dichlorofluorescein diacetate (DCFH-DA), propidium iodide (PI), chlorpromazine, dynasore and dihydrorhodamine 123 (DHR123) were purchased from Sigma-Aldrich (Shanghai, China). ATP assay kit, hypoxia-inducible factor-1 $\alpha$ (HIF-1 $\alpha$ ) ELISA kit, Ki-67 antibody staining kit and 5, 5', 6, 6'-tetrachloro-1, 1', 3, 3'tetraethylbenzimidazolylcarbocyanine iodide (JC-1) staining kit were obtained from Beyotime Biotechnology (Haimen, China).

Characterization. The hydrodynamic sizes and surface potentials of nanomaterials were determined on a Zetasizer Nano-ZS ZEN3690 instrument (Malvern, UK). Valence band XPS spectra and X-ray photoelectron spectroscopy (XPS) were recorded using Thermo ESCALAB 250Xi XPS spectrometer. The UV-vis absorbance spectra were measured using a Cary $50 \mathrm{UV}$-vis spectrometer (Varian, USA). UV-vis diffuse reflectance measurement was performed with Cary 7000 UV-vis spectrometer (Varian, USA). Fluorescence spectra were recorded on a PerkinElmer LS 55 spectrofluorometer. Powder X-ray diffraction (XRD) patterns were collected by a Rigaku Smartlab X-ray diffractometer under $\mathrm{Cu}-\mathrm{K} \alpha$ radiation $(\lambda=1.54 \AA)$. Transmission electron microscope (TEM) images and element mapping images were acquired by a JEOL-2100 transmission electron microscope with $200 \mathrm{kV}$ voltage. Fourier transform infrared (FTIR) spectra were detected by a Shimadzu FTIR-8400S spectrometer. Electron spin resonance (ESR) spectra were measured by a Bruker EMX spectrometer. The 
photocurrent response was measured on an electrochemical workstation (CHI 730E, Chenhua, China) with $650 \mathrm{~nm}$ laser as the light source using modified glassy carbon electrode (GCE) as the working electrode, $\mathrm{Ag} / \mathrm{AgCl}$ electrode as a reference electrode and platinum wire as a counter electrode, respectively. The electrochemical impedance spectroscopy (EIS) was executed on a Parstat 2273 electrochemical system (Ametek, USA). A dissolved oxygen meter (JPBJ-608, Leici, China) was used to detect $\mathrm{O}_{2}$ content in solution. The amount of $\mathrm{Au}$ was determined by an inductively coupled plasma-atomic emission spectroscopy (ICP-AES, Thermo Scientific, USA). The 650 $\mathrm{nm}$ laser (Changchun Laser Optoelectronics) was used as visible light source for photocatalytic reaction study. Confocal microscopy images were observed via a FV1000 confocal laser scanning microscopy (CLSM, Olympus, Japan). Flow analysis for cell apoptosis was carried out on a BD LSRFortessa flow cytometry (Becton, Dickinson and Company, USA). 

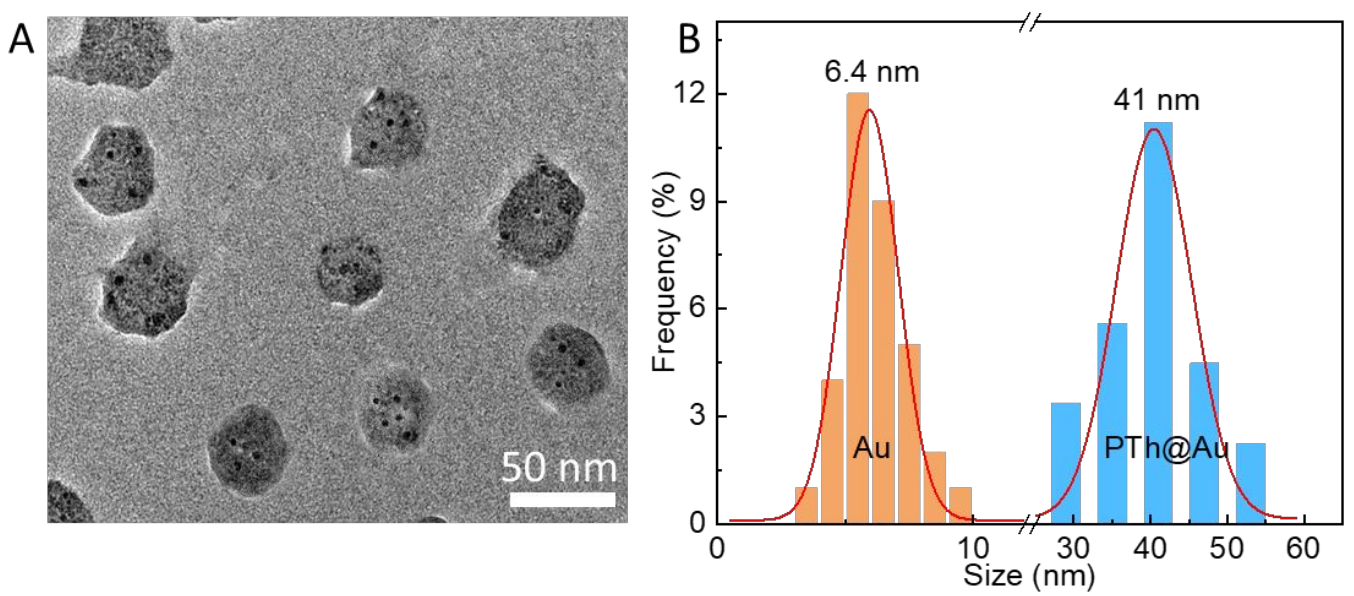

Figure S1. (A) The magnified TEM images of PTh@Au NCs. (B) Size distributions of PTh@Au NCs and Au NPs.

Furthermore, to clearly determine Au NPs size on the surface of PTh, the magnified TEM image of PTh@Au NCs surface displayed that Au NPs with $~ 6.4 \mathrm{~nm}$ were formed on the surface of PTh (Figure S1A), which well agreed with HRTEM image in Figure 1B. 


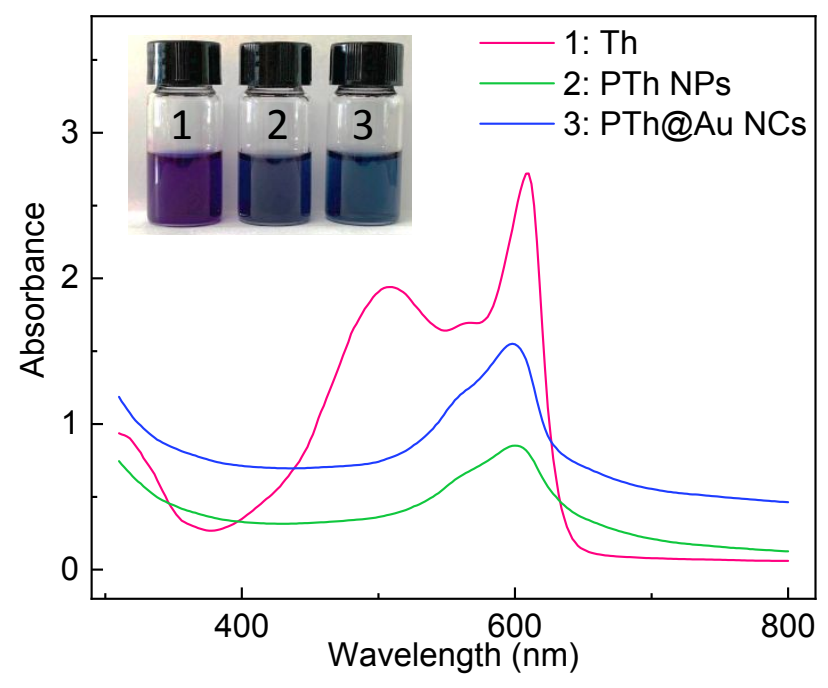

Figure S2. UV-vis spectra of Th, PTh NPs and PTh@Au NCs. Inset is a digital photo of Th, PTh NPs and PTh@Au NCs dispersed in DMF.

Thionine as a phenothiazine dye tends to accumulate in aqueous solution with dimerization constant of $5.6 \times 10^{2} \mathrm{M}^{-1}$. Therefore, we compare absorption spectra of the free thionine with polythionine in DMF. The monomer band at $509 \mathrm{~nm}$ can only be clearly observed in free thionine solution, which has been also reported in previous works. ${ }^{1}$ Meanwhile, the spectra of thionine also exhibited a characteristic maximum at $610 \mathrm{~nm}$ with a shoulder at $570 \mathrm{~nm}$ attributed previously to J-aggregates $(610 \mathrm{~nm})$ and H-type dimers of the dye $(570 \mathrm{~nm}),{ }^{2}$ regardless of the concentration used, and only upon very strong dilution of the solution, one can see the monomer band (509 $\mathrm{nm})$, being all the time in equilibrium with the aggregated form. However, the absorption peak at 509 $\mathrm{nm}$ disappeared by the occurrence of polymerization, which was also confirmed by the difference of exterior color of thionine (purple) and PTh (ink blue). The distinguishable color showed the structure of polythionine was possibly different from thionine. 


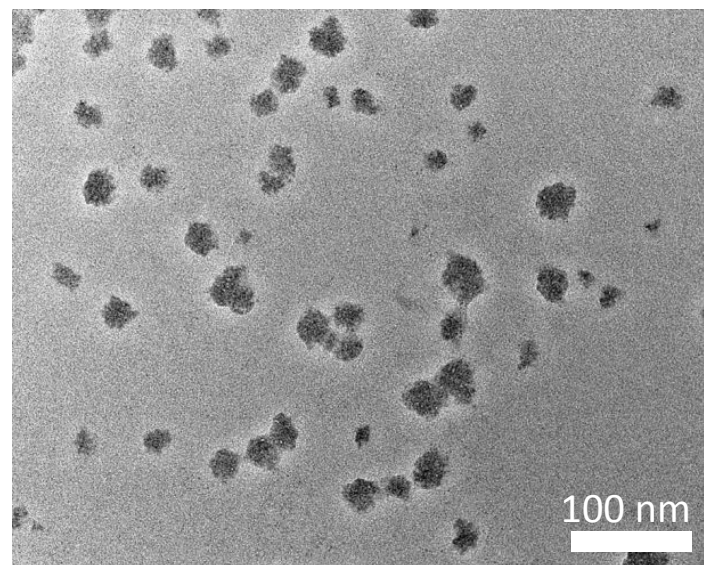

Figure S3. TEM image of PTh NPs. 
<smiles></smiles>

Thionine

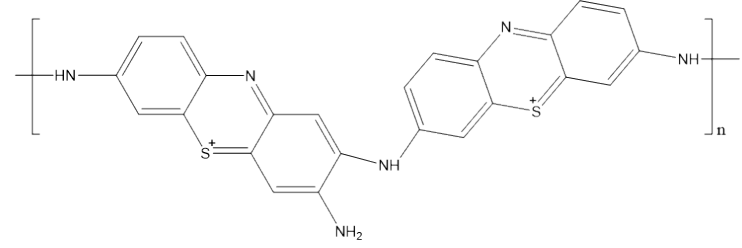

Polythionine

Figure S4. The proposed polymerization process of thionine. 


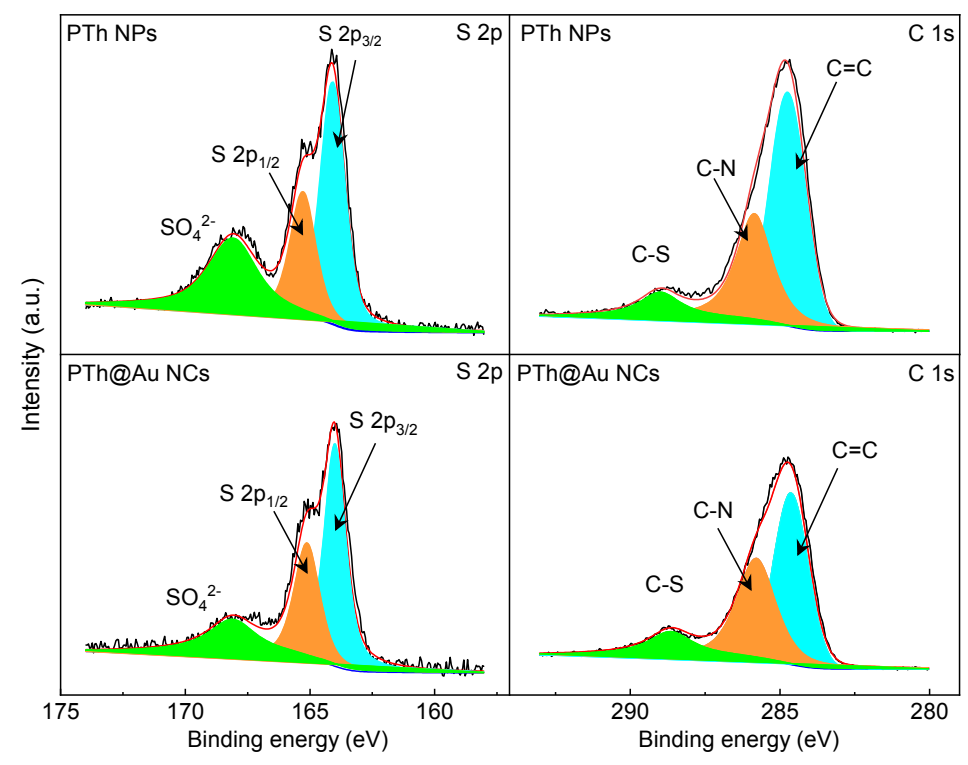

Figure S5. S 2p and C 1s high-resolution XPS spectra of the PTh NPs and PTh@Au NCs.

The XPS pattern of PTh@Au NCs showed the coexistence of C, S, N, O and Au signals corresponding to characteristic peaks at 285.7, 163.7, 399.6, 532.7 and $85.5 \mathrm{eV}$, respectively, confirming the successful incorporation of Au in PTh@Au NCs (Figure 1E). The high-resolution S spectra of pure PTh NPs demonstrated three characteristic peaks at $164.1 \mathrm{eV}, 165.3 \mathrm{eV}$, and $168.1 \mathrm{eV}$ assigned to $\mathrm{S} 2 \mathrm{p}_{3 / 2}, \mathrm{~S} 2 \mathrm{p}_{1 / 2}$ and $\mathrm{SO}_{4}{ }^{2-}$, respectively. In comparison with the $S 2 p$ spectra in pure PTh NPs, the peaks of $S 2 p$ (163.9 eV of S 2 $p_{3 / 2}$ and 165.0 eV of S 2 $p_{1 / 2}$ ) in PTh@Au NCs were shifted to lower binding energy with the weaker intensity, which again indicated the existence of sulfur vacancies (Figure S5). Meanwhile, the sulfur vacancies also induced the three peaks of C1s spectra (284.6 eV of C=C, 285.7 eV of C-N and 288.6 eV of C-S) in PTh@Au NCs to shift to lower binding energy compared with that in PTh NPs (284.8, 285.8 and 289.0 eV, Figure S5).

Table S1. The elemental compositions for PTh@Au NCs and PTh NPs determined by XPS. Results were expressed in atom \%.

\begin{tabular}{ccccccc}
\hline Sample & $\mathrm{C}$ & $\mathrm{S}$ & $\mathrm{N}$ & $\mathrm{O}$ & $\mathrm{Fe}$ & $\mathrm{Au}$ \\
\hline PTh@Au & 74.13 & 3.48 & 9.27 & 12.51 & 0.26 & 0.35 \\
PTh & 73.71 & 3.79 & 8.6 & 13.56 & 0.34 & $/$ \\
\hline
\end{tabular}




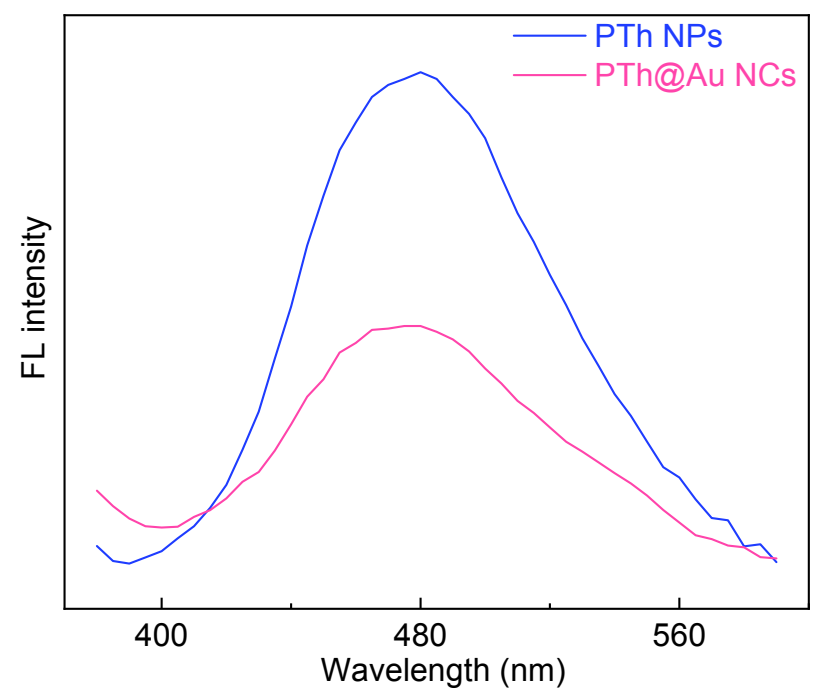

Figure S6. Fluorescence spectra of the PTh NPs and PTh@Au NCs. 

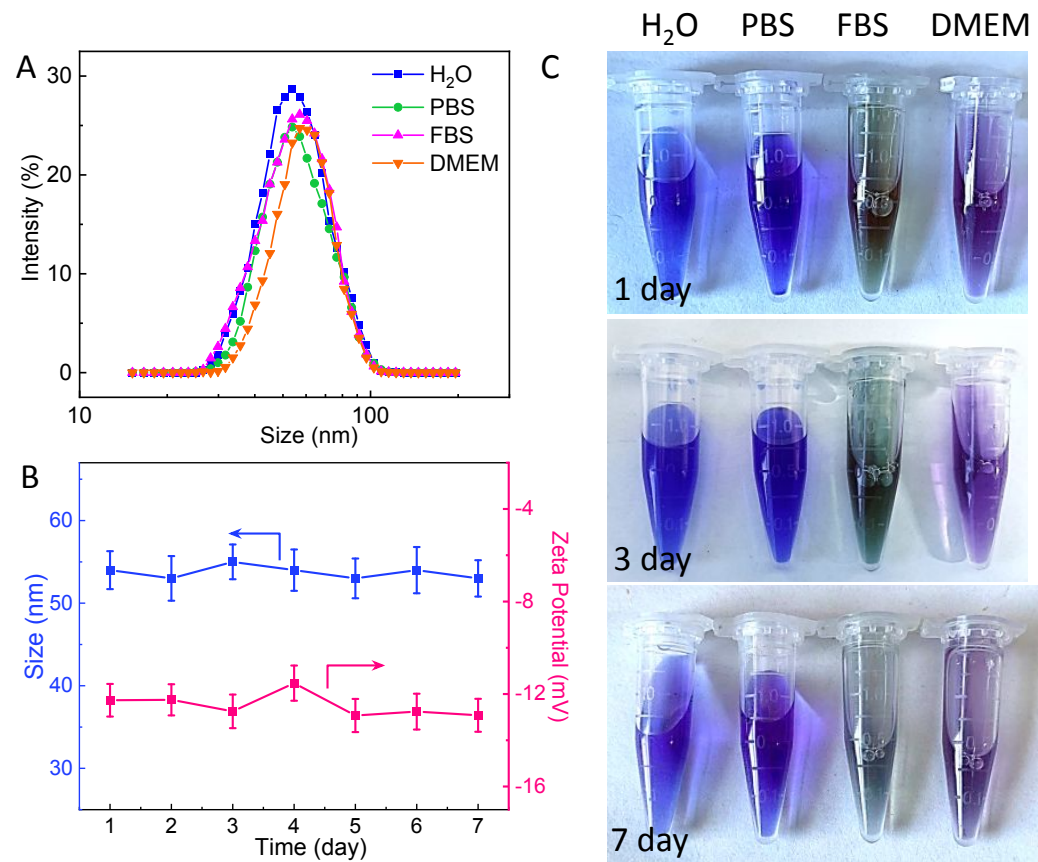

Figure S7. (A) Hydrodynamic diameter distributions of PTh@Au NCs in $\mathrm{H}_{2} \mathrm{O}$ (PDI: 0.13), PBS (PDI: 0.17), FBS (PDI: 0.14) and DMEM (PDI: 0.16). (B) The variation of hydrodynamic sizes and zeta potentials of PTh@Au NCs in one week. (C) The photographs of PTh@Au NCs dispersed in $\mathrm{H}_{2} \mathrm{O}$, PBS, FBS and DMEM cell-culture medium at 1, 3 and 7 day, respectively.

The obtained PTh@Au NCs exhibits excellent dispersion with imperceptible size variation in different physiological environments (Figure S7A), such as water, phosphate buffered solution (PBS), fetal bovine serum (FBS), and dulbecco's modified eagle medium (DMEM). All the polydispersity index (PDI) value of PTh@Au NCs in different physiological environments is less than 0.2 , indicating a nearly monodisperse size distribution. 


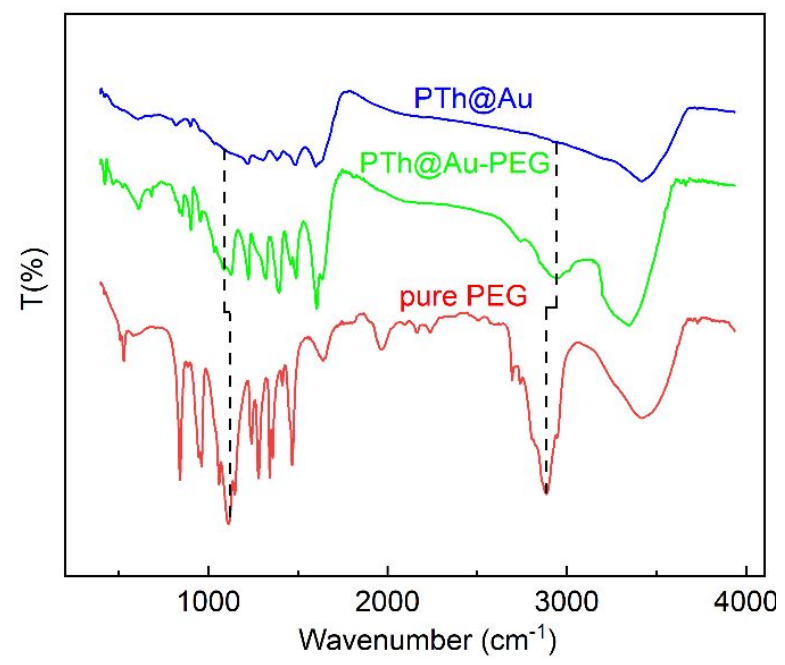

Figure S8. FTIR spectra PTh@Au, PTh@Au-PEG and pure PEG.

In addition, the attachment of PEG on the PTh@Au was verified by FTIR analysis. The peak around $2882 \mathrm{~cm}^{-1}$ is attributed to the alkyl chain of PEG; another band at $1114 \mathrm{~cm}^{-1}$ belonged to the $\mathrm{C}-\mathrm{O}-\mathrm{C}$ stretching vibration. These two peaks are the characteristic adsorption bands of PEG, which are also demonstrated and shifted to 2936 and $1083 \mathrm{~cm}^{-1}$ in PTh@Au-PEG, suggesting the successful attachment of PEG to PTh@Au. 

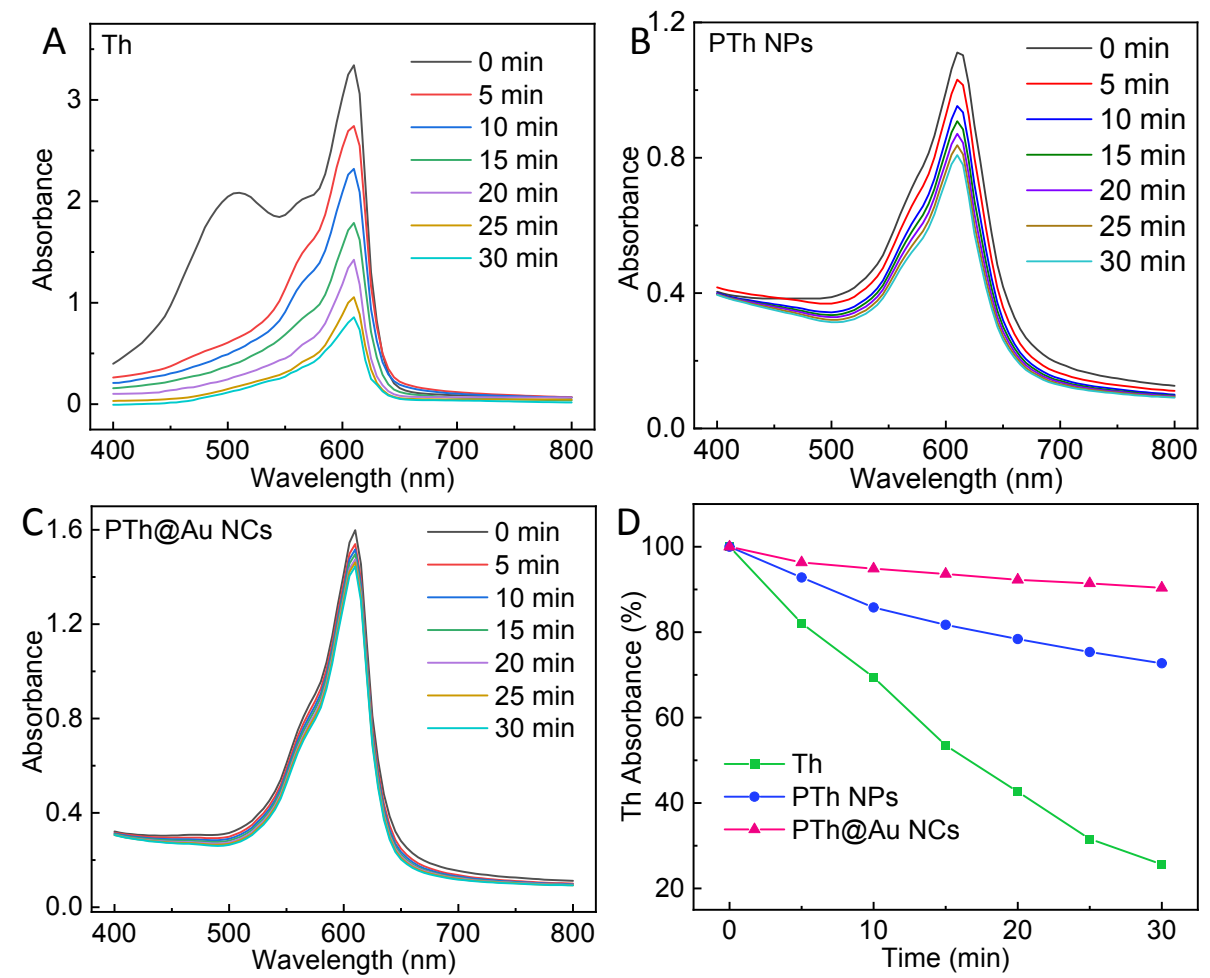

Figure S9. The UV-Vis spectra of free Th (A), PTh NPs (B) and PTh@Au NCs (C) over laser irradiation time. (D) Relative absorbance changes of Th, PTh NPs and PTh@Au NCs over laser irradiation time. 

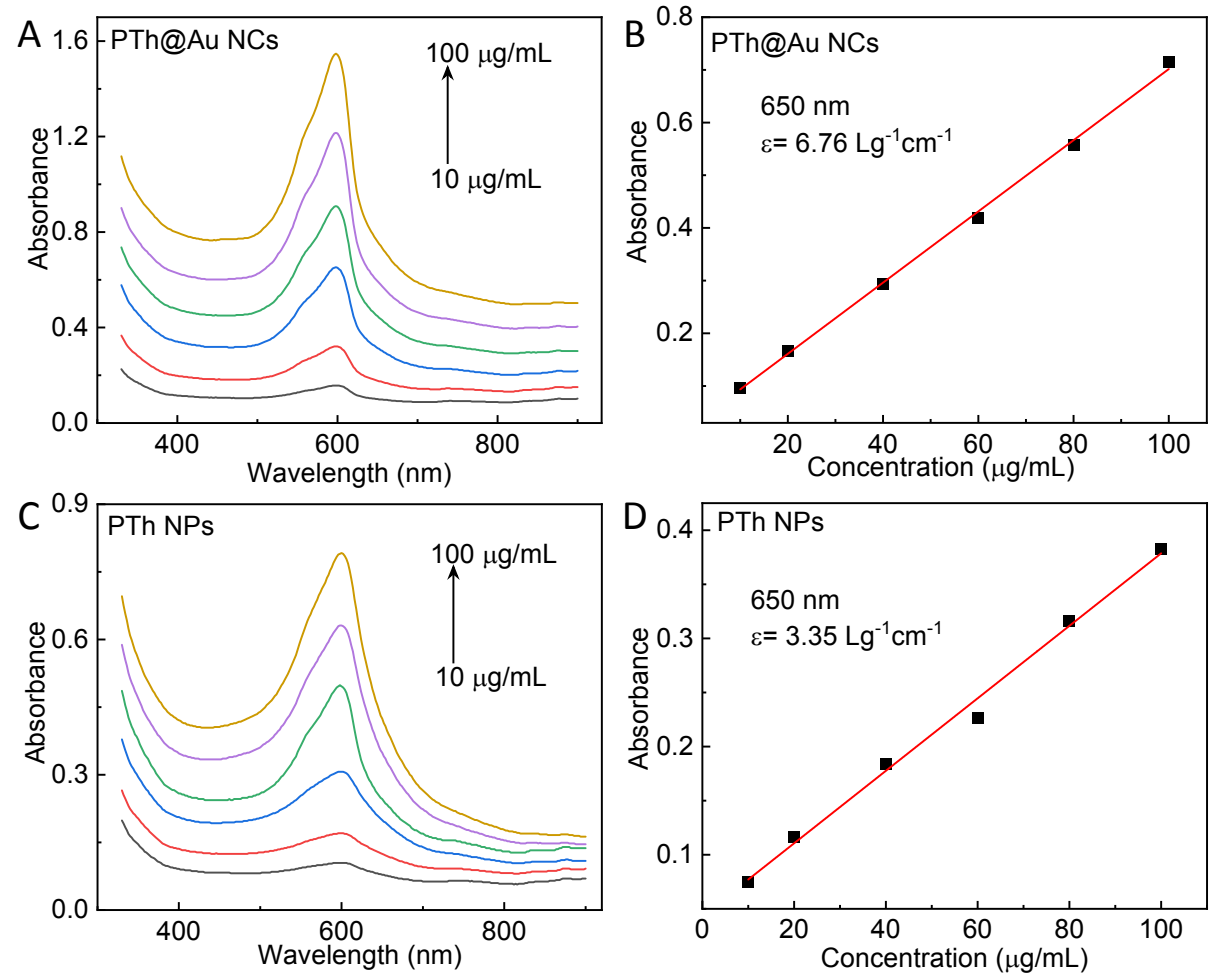

Figure S10. The UV-vis spectra of different concentrations of the PTh@Au NCs (A) and PTh NPs (C). Mass extinction coefficient of the PTh@Au NCs (B) and PTh NPs (D) at $650 \mathrm{~nm}$.

The mass extinction coefficient $(\varepsilon)$ at $650 \mathrm{~nm}$ was measured to evaluate the lightabsorption capability. Based on the Lambert-Beer law, ${ }^{3}$ the $\varepsilon$ was calculated according to the following equation:

$A=\varepsilon L C$

$A$ : the absorbance of materials at $650 \mathrm{~nm}$.

$\varepsilon$ : the mass extinction coefficient $\left(\mathrm{L} \mathrm{g} \mathrm{g}^{-1} \mathrm{~cm}^{-1}\right)$.

$L$ : the path length of $1 \mathrm{~cm}$.

$C$ : the concentration of materials $\left(\mathrm{g} \mathrm{L}^{-1}\right)$.

Then, we can obtain: $A / L=\varepsilon C$. The slope of $A / L v s$. concentration of $C$ was the mass extinction coefficient of $\varepsilon$, which was estimated to be $6.76 \mathrm{~L} \mathrm{~g}^{-1} \mathrm{~cm}^{-1}$ and $3.35 \mathrm{~L} \mathrm{~g}^{-1}$ $\mathrm{cm}^{-1}$ for PTh@Au NCs and PTh NPs, respectively. 


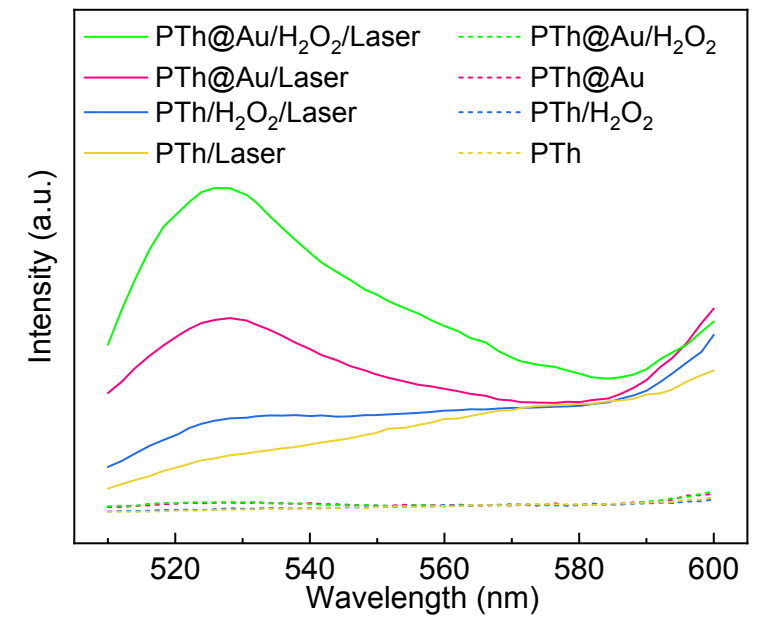

Figure S11. Fluorescence spectra of DCFH in PTh@Au/ $\mathrm{H}_{2} \mathrm{O}_{2}, \mathrm{PTh} @ \mathrm{Au}, \mathrm{PTh} / \mathrm{H}_{2} \mathrm{O}_{2}$ and PTh dispersions with or without a $650 \mathrm{~nm}$ laser irradiation for $30 \mathrm{~min}$. 

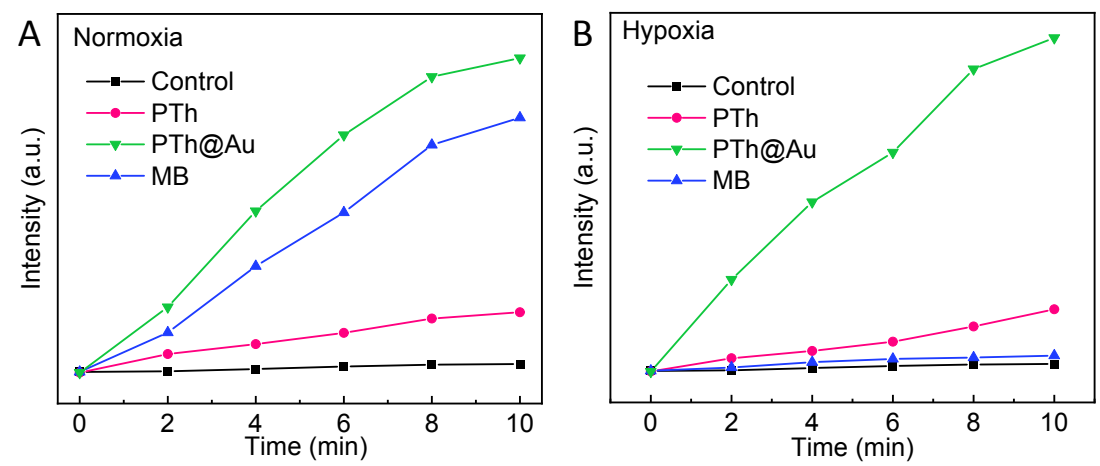

Figure S12. The time-dependent fluorescence changes of SOSG at $530 \mathrm{~nm}$ due to ${ }^{1} \mathrm{O}_{2}$ generation from PBS, PTh NPs, PTh@Au NCs and MB in normoxia (A) or hypoxia (B) conditions with laser irradiation. 


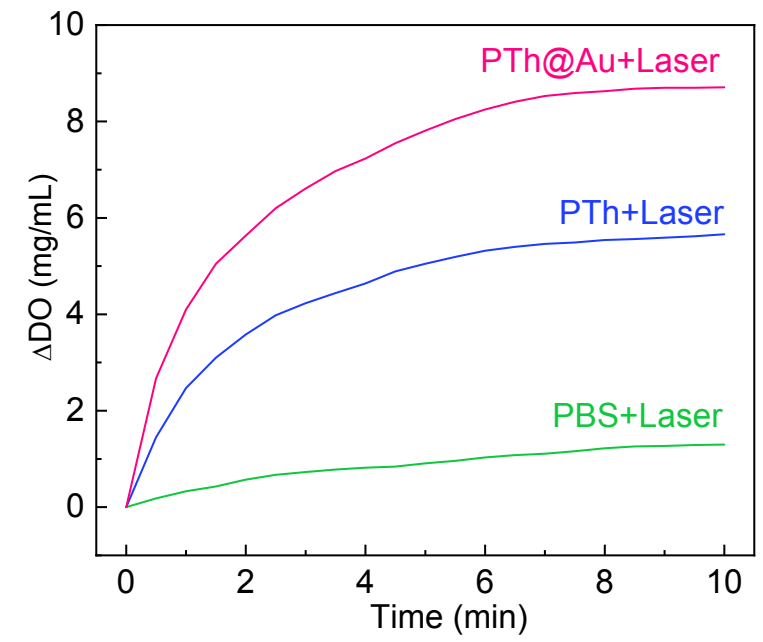

Figure S13. $\mathrm{O}_{2}$ generation profiles in PBS, PTh NPs and PTh@Au NCs dispersions with a $650 \mathrm{~nm}$ laser irradiation for $10 \mathrm{~min}$. 


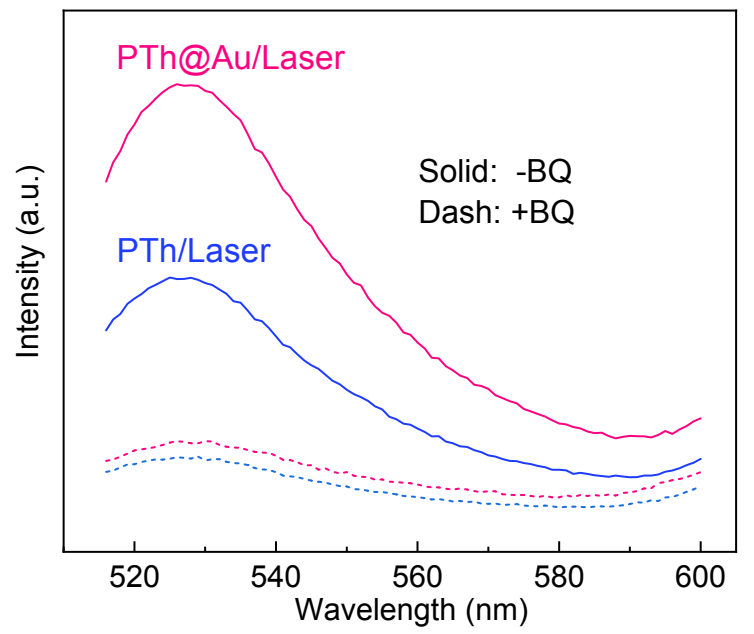

Figure S14. Fluorescence spectra of DHR123 in PTh@Au NCs and PTh NPs dispersions after irradiation with a $650 \mathrm{~nm}$ laser in absence or presence of $\mathrm{BQ}$. 


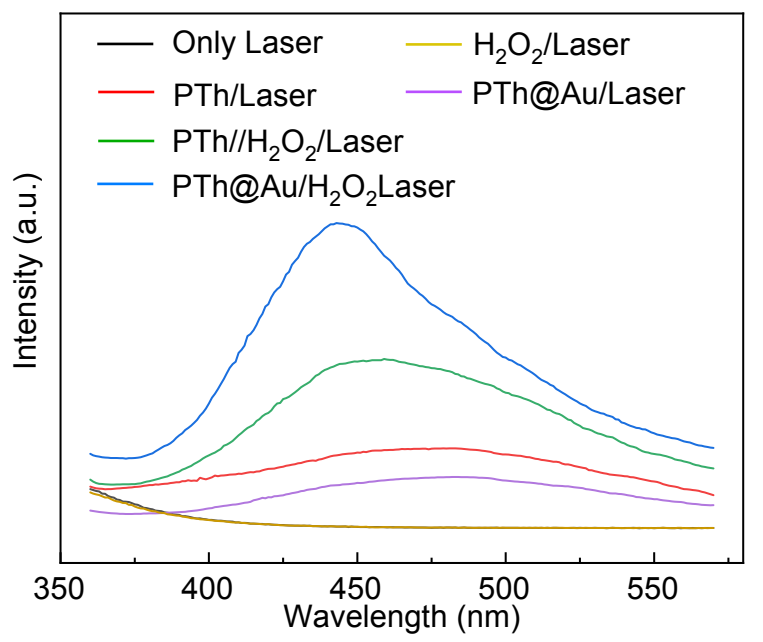

Figure S15. Fluorescence spectra of TA in PBS, PTh NPs and PTh@Au NCs dispersions after irradiation with a $650 \mathrm{~nm}$ laser in absence or presence of $\mathrm{H}_{2} \mathrm{O}_{2}$. 

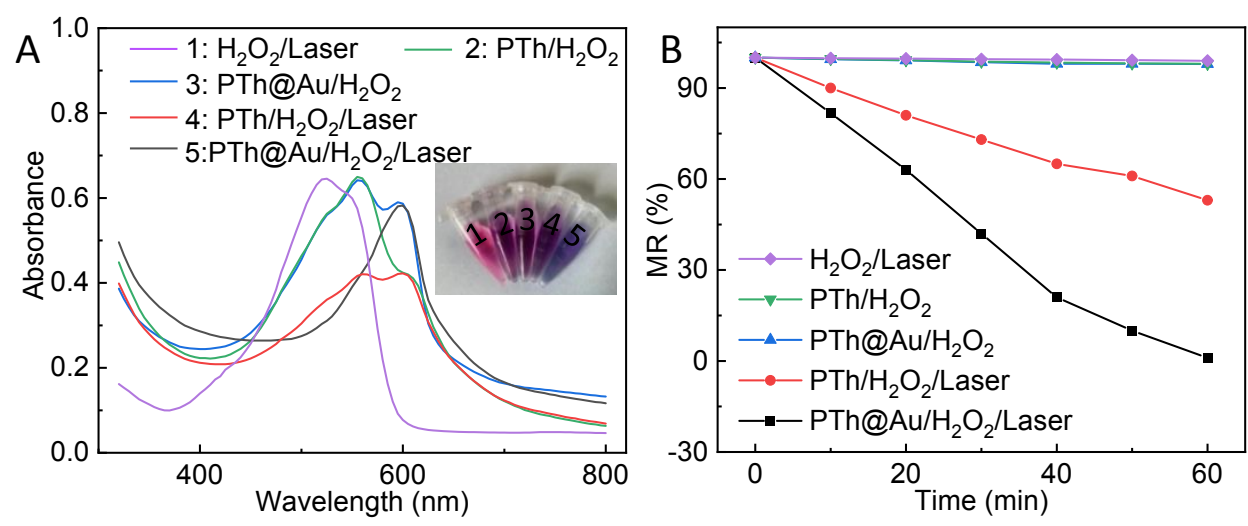

Figure S16. (A) The UV-vis spectra of MR after different treatments. Inset: digital photos demonstrated the color contrast of MR after different treatments. (B) Photodegradation of MR under irradiation of $650 \mathrm{~nm}$ with various treatments. 


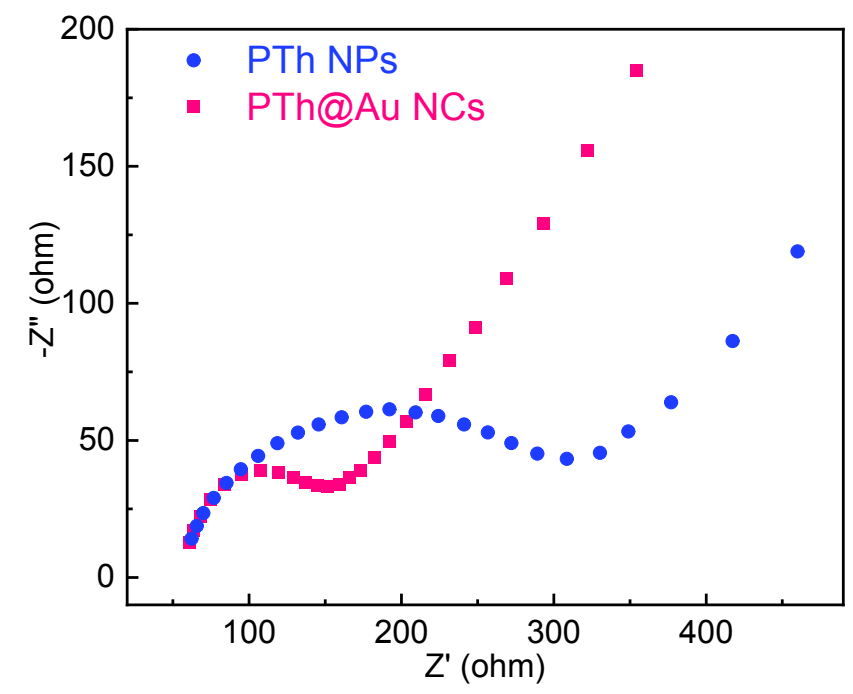

Figure S17. EIS plots of PTh NPs and PTh@Au NCs.

To better clarify the charge transfer resistance of PTh@Au NCs, electrochemical impedance spectroscopy measurements were carried out, and both of the Nyquist plots were composed of a small pseudo-semicircle arc at higher frequency range and an inclined line at lower frequency range. The diameter of the semicircle arc was related to the charge-transfer resistance $\left(R_{\mathrm{ct}}\right)$. The equivalent circuit fitting results revealed that the charge-transfer resistance $\left(R_{\mathrm{ct}}\right)$ was decreased from $261.4 \Omega$ to $92.9 \Omega$ after Auincorporation in PTh (Figure S17), suggesting that the Au-incorporation can significantly decrease the charge transfer resistance of PTh@Au NCs. 

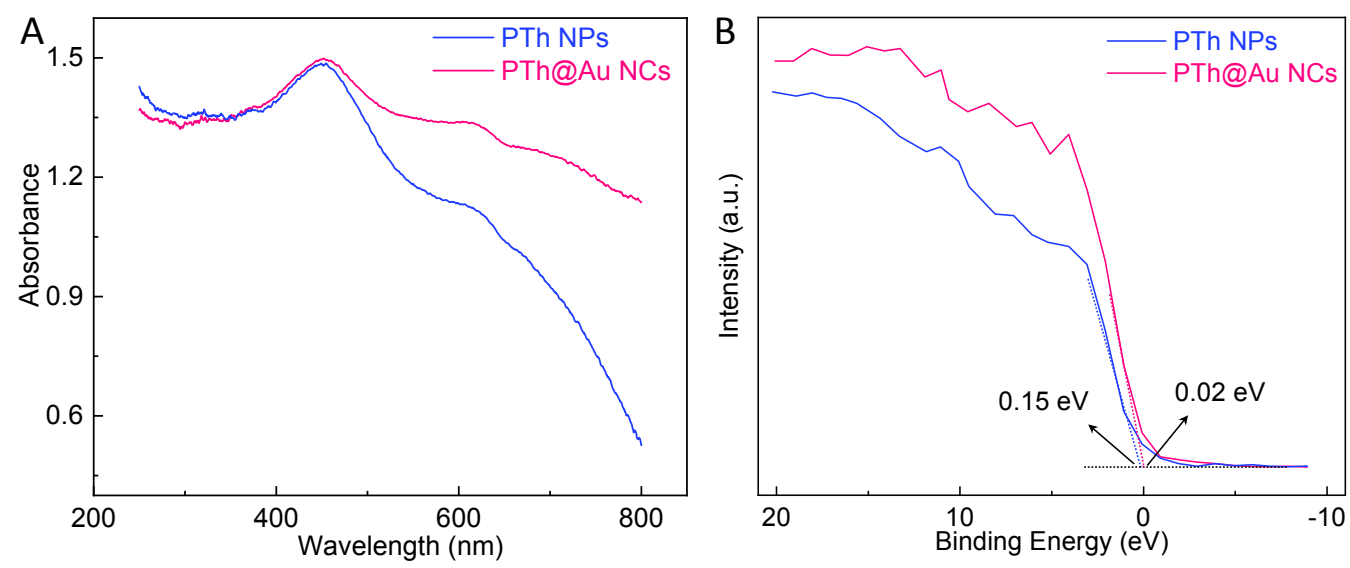

Figure S18. Diffuse reflectance spectra (A) and VB-XPS spectra (B) of PTh NPs and PTh@Au NCs.

The optical band gap energies of PTh@Au NCs and PTh NPs were evaluated by UV-vis diffuse reflectance spectroscopy, according to the following equation: $\alpha h v=$ $A\left(h v-E_{\mathrm{g}}\right)^{\mathrm{n}}$, where $\alpha, E_{\mathrm{g}}, v$ and $h$ represent the absorbance of materials, band gap energies, photon frequency, and Planck's constant. ${ }^{4}$ According to Figure $2 \mathrm{H}$, the band gap energies $\left(E_{\mathrm{g}}\right)$ of PTh@Au NCs and PTh NPs were $1.80 \mathrm{eV}$ and $2.00 \mathrm{eV}$, respectively, which were responsive to light of $650 \mathrm{~nm}(2.006 \mathrm{eV})$. The valence bandedge potentials (VB) of PTh@Au NCs and PTh NPs were measured as $0.02 \mathrm{eV}$ and $0.15 \mathrm{eV}$, respectively, from the VB-XPS plots. The VB values of PTh@Au NCs and PTh NPs $v s$. NHE were calculated by the empirical equation: $E_{\mathrm{NHE}}=\varphi+E_{\mathrm{VB}-\mathrm{XPS}}-4.44$, $E_{\mathrm{NHE}}$ is standard hydrogen electrode potential; $E_{\mathrm{VB}-\mathrm{XPS}}$ is VB value tested by VB-XPS; $\varphi$ is electron work function of the XPS analyzer $(4.55 \mathrm{eV})$, and the VB values vs. NHE were calculated to be $0.13 \mathrm{eV}$ and $0.26 \mathrm{eV}$ for PTh@Au NCs and PTh NPs, respectively. The conduction band $(\mathrm{CB})$ position can be determined by $E_{\mathrm{CB}}=E_{\mathrm{VB}}-E_{\mathrm{g}}$, and $E_{\mathrm{CB}}$ was calculated to be $-1.67 \mathrm{eV}$ and $-1.74 \mathrm{eV}$ for PTh@Au NCs and PTh NPs, respectively. 

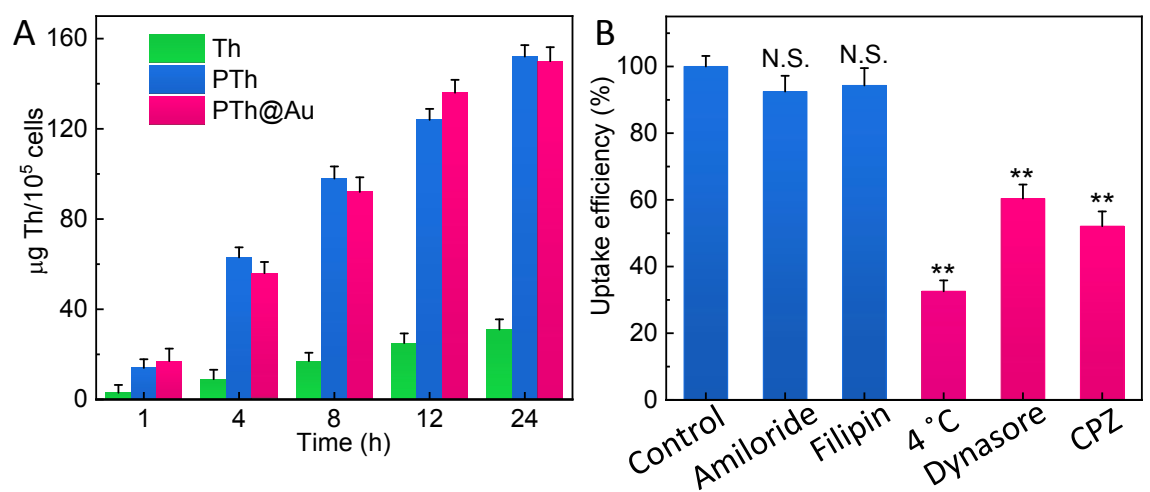

Figure S19. (A) Cellular uptake of Th, PTh NPs and PTh@Au NCs by HeLa cells. (B) Uptake efficiency of PTh@Au NCs by HeLa cells treated by amiloride, filipin, dynasore, or CPZ for $1 \mathrm{~h}$ at $37^{\circ} \mathrm{C}$ or $4^{\circ} \mathrm{C}$. Statistical significance was determined by Student's t test. $p>0.05$ (N.S., no significant difference), ${ }^{*} p<0.05, * * p<0.01,{ }^{* * *} p$ $<0.001$.

Cellular uptake of materials was quantified by Th content per cell through UV-vis absorbance spectra detection at $600 \mathrm{~nm}$. After incubated with free Th, PTh NPs and PTh@Au NCs for different times, the absorbance signals of Th inside the HeLa cells increased gradually with prolonging the incubation time (Figure S19A). The cellular uptake amounts of PTh NPs $\left(\sim 38.0 \mu \mathrm{g}\right.$ Th/10 $0^{5}$ cells $)$ and PTh@Au NCs $(\sim 37.5 \mu \mathrm{g}$ $\mathrm{Th} / 10^{5}$ cells $)$ were approximately 5.0 times higher than that of free $\mathrm{Th}\left(\sim 7.75 \mu \mathrm{g} \mathrm{Th} / 10^{5}\right.$ cells) after 24 h incubation, indicating the high endocytosis of PTh NPs and PTh@Au NCs.

To further investigate the endocytosis pathway, the HeLa cells were incubated at low temperature $\left(4^{\circ} \mathrm{C}\right)$ or with clathrin inhibitor (chlorpromazine, $\mathrm{CPZ}$ ) and dynamin inhibitor (dynasore) to determine whether the cellular uptake of PTh@Au was energy dependent. The results showed that the cellular uptake of PTh@Au NCs was significantly inhibited compared with control cells (Fig. S19B). However, the cellular uptake of PTh@Au NCs was not inhibited by caveolae inhibitor (filipin) or macropinocytosis inhibitor (amiloride). These results demonstrated that the PTh@Au NCs can be effectively internalized into cells through energy-dependent endocytosis process mediated by clathrin. 


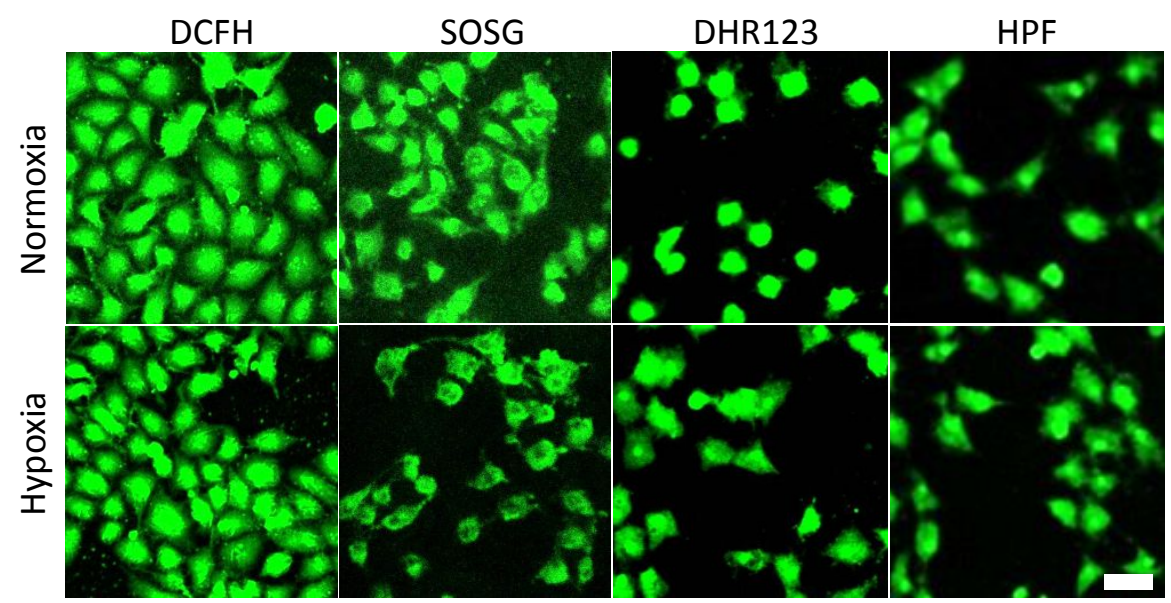

Figure S20. CLSM images of HeLa cells stained with DCFH, SOSG, DHR123 and HPF in hypoxia and normoxia conditions with PTh@Au NCs treatment. Scale bar: 50 $\mu \mathrm{m}$. 


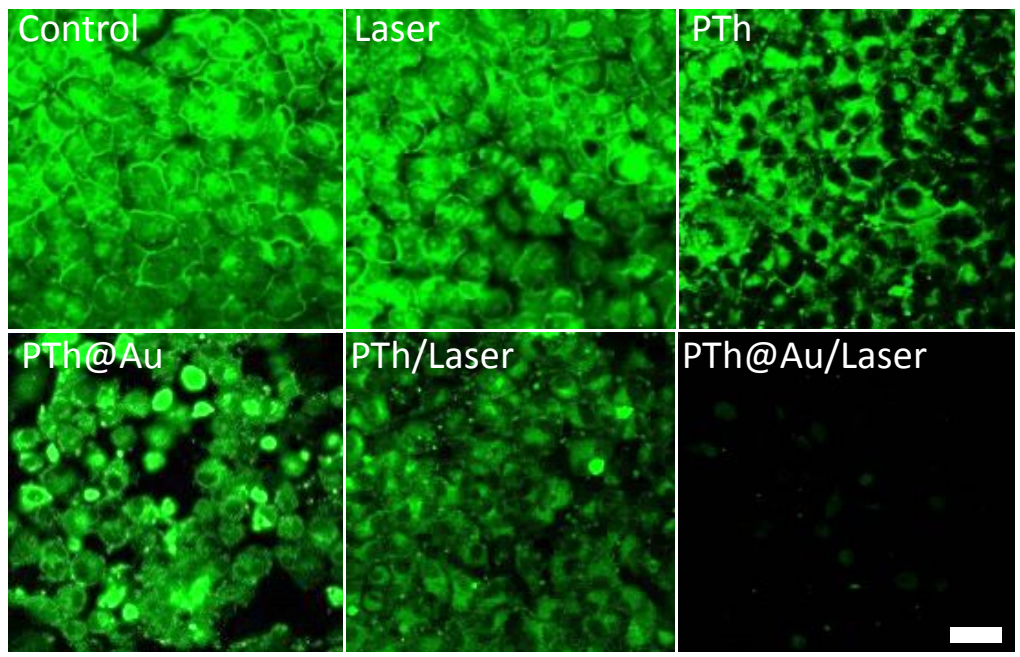

Figure S21. CLSM images of HeLa cells stained with $\mathrm{O}_{2}$ probe of RDPP after different treatments. Scale bar: $50 \mu \mathrm{m}$. 

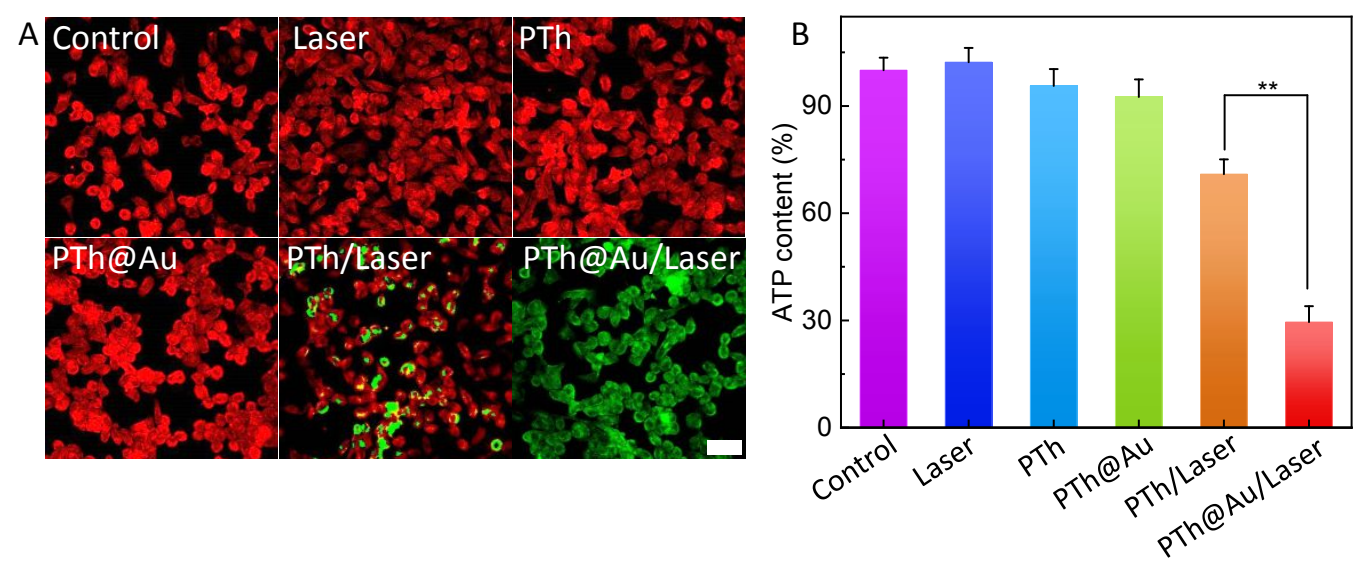

Figure S22. (A) CLSM images of HeLa cells stained with JC-1 after different treatments. (B) The intracellular ATP level after different treatments. Scale bar: $50 \mu \mathrm{m}$. Statistical significance is determined by Student's t test. ${ }^{*} p<0.05,{ }^{* *} p<0.01,{ }^{* * *} p<$ 0.001 . 
A

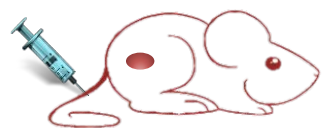

Nanoconstructs Injection (Day -1/-2)
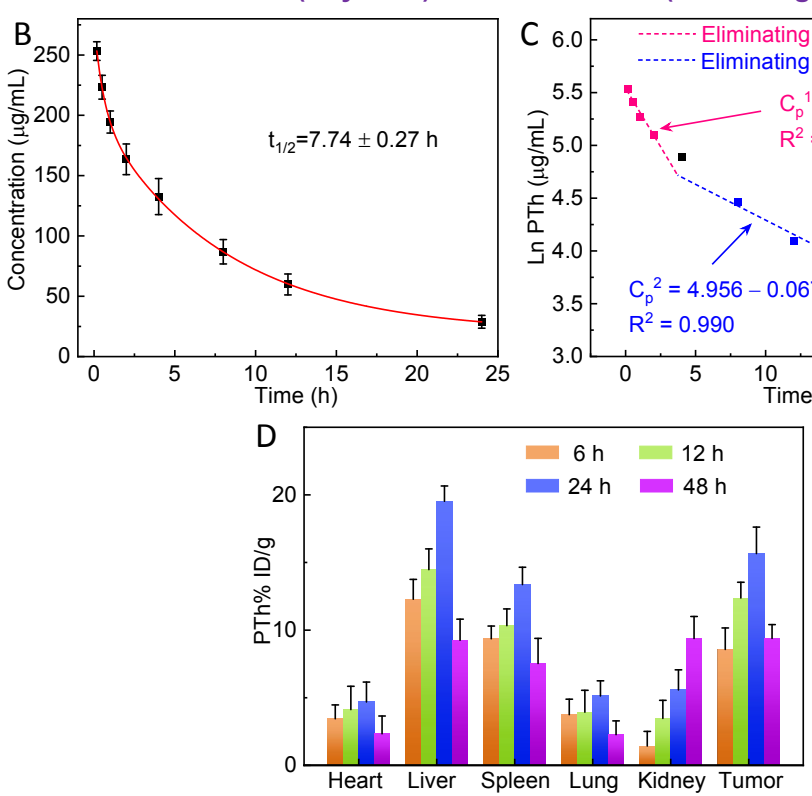

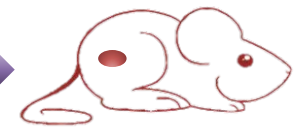

Pharmacokinetics Evaluation (Blood/Organs)

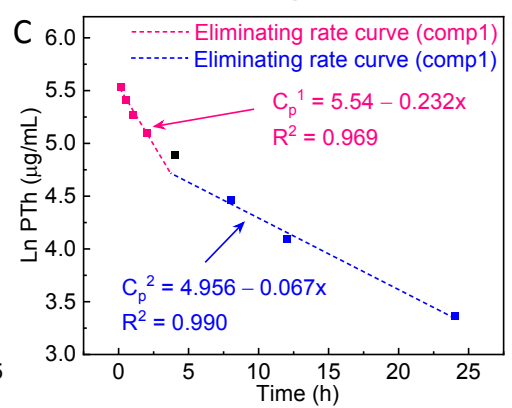

$$
\text { (3) }
$$


A

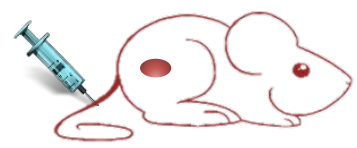

Nanoconstructs Injection (Day -14)
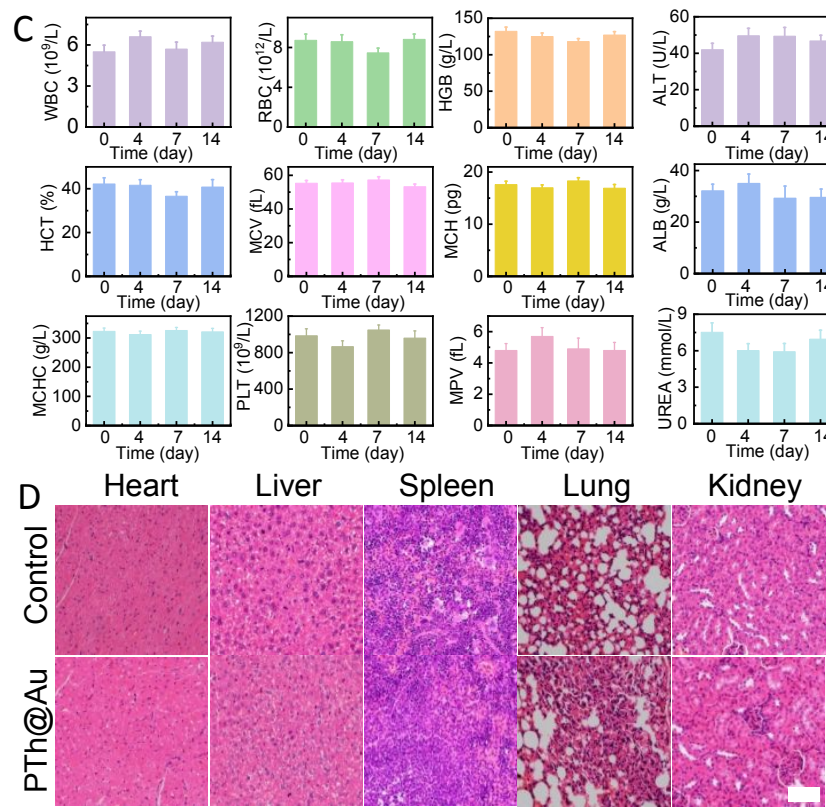

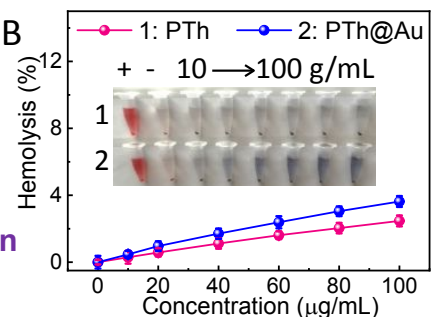

(Blood/Organs)
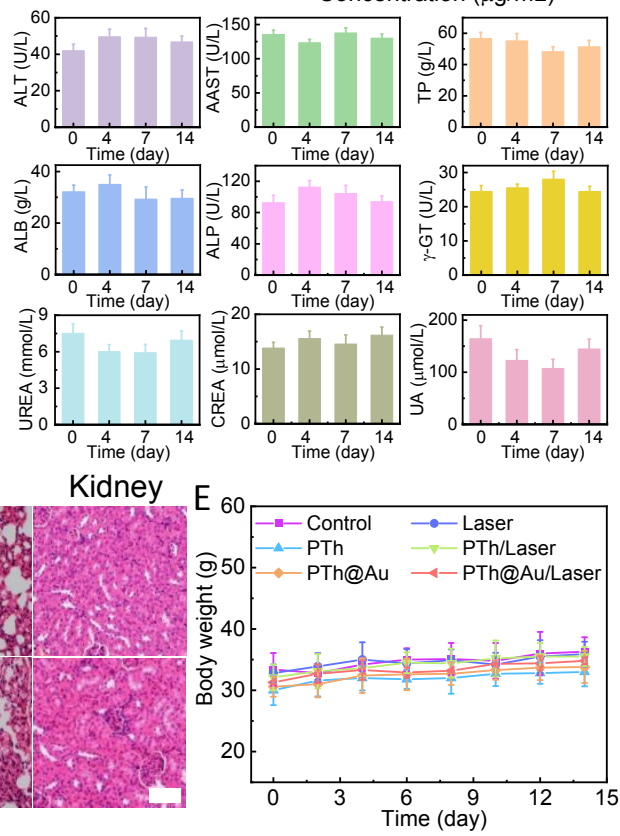

Figure S24. (A) Schematic illustration of the biosafety assessment of PTh@Au NCs in tumor-bearing mice. (B) Hemolysis tests for the PTh NPs and PTh@Au NCs with disparate concentrations. (C) Blood routine and biochemistry examination for the mice treated with the PTh@Au NCs at 0,4, 7 and 14 day. (D) H\&E staining of major organs from mice treated with or without PTh@Au/Laser. (E) Body weight curves of the mice after various treatments. 
Table S2. Summary of therapy effectiveness of photodynamic platforms

\begin{tabular}{|c|c|c|c|c|}
\hline Category & Tumor types & $\begin{array}{l}\text { Therapeutic } \\
\text { mechanisms }\end{array}$ & $\begin{array}{l}\text { Tumor } \\
\text { inhibition } \\
\text { rates }\end{array}$ & Ref. \\
\hline $\begin{array}{l}\mathrm{CaCO}_{3} @ \mathrm{COF}- \\
\text { BODIPY-2I@GAG }\end{array}$ & $\begin{array}{l}\text { HCT-116 } \\
\text { tumor models }\end{array}$ & $\begin{array}{lr}\text { Light-triggered } & { }^{1} \mathrm{O}_{2} \\
\text { and }{ }^{1} \mathrm{O}_{2} \text {-triggered } \\
\mathrm{Ca}^{2+} & \text { overload } \\
\text { enhanced } & \text { PDT } \\
\text { efficiency } & \end{array}$ & $90.6 \%$ & 5 \\
\hline $\begin{array}{l}\text { PFD@COFTTA- } \\
\text { DHTA@PLGA-PEG }\end{array}$ & $\begin{array}{c}\text { CT26 } \\
\text { tumor models }\end{array}$ & $\begin{array}{l}\text { The alleviated tumor } \\
\text { hypoxia and } \\
\text { improved tumor } \\
\text { homing of } \\
\text { photosensitizer } \\
\text { increase the ROS } \\
\text { generation }\end{array}$ & $92 \%$ & 6 \\
\hline $\begin{array}{l}\text { Polymer conjugated } \\
\text { indocyanine green and } \\
\text { photosensitizer chlorin } \\
\text { e6 }\end{array}$ & $\begin{array}{c}4 \mathrm{~T} 1 \\
\text { tumor models }\end{array}$ & $\begin{array}{l}\text { The singlet oxygen } \\
\text { produced by Ce6 can } \\
\text { ablate the tumor cells } \\
\text { in the normoxic } \\
\text { microenvironment }\end{array}$ & $80.7 \%$ & 7 \\
\hline $\begin{array}{l}\text { Porphyrin/camptothec } \\
\text { in-floxuridine triad } \\
\text { microbubbles (PCF- } \\
\text { MBs) }\end{array}$ & $\begin{array}{c}\text { HT-29 } \\
\text { tumor models }\end{array}$ & $\begin{array}{l}\text { Porphyrin-induced } \\
{ }^{1} \mathrm{O}_{2} \text { production under } \\
\text { light irradiation and } \\
\text { improved drug uptake } \\
\text { caused severe } \\
\text { cytotoxicity }\end{array}$ & $90 \%$ & 8 \\
\hline $\begin{array}{l}\text { Tumor-targeted hybrid } \\
\text { protein } \\
\text { carriers } \\
\text { encapsulation } \\
\text { doxorubicin }(\mathrm{HPOCs}) \\
\text { and chlorin e6 }(\mathrm{Ce} 6)\end{array}$ & $\begin{array}{c}\text { MCF-7 } \\
\text { tumor models }\end{array}$ & $\begin{array}{l}\text { Sufficient oxygen } \\
\text { supply enhance ROS } \\
\text { generation of PS }\end{array}$ & $89.5 \%$ & 9 \\
\hline $\begin{array}{l}\mathrm{ZnF}_{16} \mathrm{Pc} \text {-loaded } \\
\text { ferritins onto red blood } \\
\text { cells }\end{array}$ & $\begin{array}{l}\text { U87MG } \\
\text { tumor models }\end{array}$ & $\begin{array}{l}\text { RBC-facilitated PDT } \\
\text { harnessing } \\
\text { erythrocytes as an } \mathrm{O}_{2} \\
\text { transporter, can } \\
\text { efficiently produce } \\
{ }^{1} \mathrm{O}_{2} \text { even under low } \\
\text { oxygen conditions }\end{array}$ & $76.7 \%$ & 10 \\
\hline
\end{tabular}


MC540-

SAO:Eu@mSiO

nanoparticles

Zinc

hexadecafluorophthal

ocyanine $\left(\mathrm{ZnF}_{16} \mathrm{Pc}\right)$

encapsulated into

RGD4C-modified

ferritin

Au-decorated

polythionine

nanoconstructs with

rich sulfur vacancies
X-ray as an energy

H1299 source was utilized to

tumor models activate a PDT

process

Ferritin as an efficient PS delivery vehicle can encapsulate a large amount of PS and selectively deliver it to tumors to induce efficient PDT

Sulfur vacancies in PTh@Au NCs, induced an efficient electron-hole separation and

$83.64 \% \quad 12$ tumor models generated a $\sim 2$-3-fold more ROS for PDT.
$54.2 \% \quad 11$

95\% This work 


\section{References}

(1) Zhao, C.; Jiang, Z.; Cai, X.; Lin, L.; Lin, X.; Weng, S., Ultrasensitive and Reliable Dopamine Sensor Based on Polythionine/Aunps Composites. J. Electroanal. Chem. 2015, 748, 16-22.

(2) Krzyszkowska, E.; Walkowiak-Kulikowska, J.; Stienen, S.; Wojcik, A., ThionineGraphene Oxide Covalent Hybrid and Its Interaction with Light. PCCP 2017, 19, 14412-14423.

(3) Feng, W.; Han, X.; Wang, R.; Gao, X.; Hu, P.; Yue, W.; Chen, Y.; Shi, J., Nanocatalysts-Augmented and Photothermal-Enhanced Tumor-Specific Sequential Nanocatalytic Therapy in Both NIR-I and NIR-II Biowindows. Adv. Mater. 2019, 31, 1805919.

(4) Zhen, W.; Liu, Y.; Jia, X.; Wu, L.; Wang, C.; Jiang, X., Reductive SurfactantAssisted One-Step Fabrication of a $\mathrm{BiOI} / \mathrm{BiOIO}_{3}$ Heterojunction Biophotocatalyst for Enhanced Photodynamic Theranostics Overcoming Tumor Hypoxia. Nanoscale Horiz. 2019, 4, 720-726.

(5) Dong, Y. B.; Guan, Q.; Zhou, L. L.; Lv, F. H.; Li, W. Y.; Li, Y. A., A Glycosylated Covalent Organic Framework Equipped with Bodipy and $\mathrm{CaCO}_{3}$ for Synergistic Tumor Therapy. Angew. Chem. Int. Ed. 2020, 59, 18042-18047.

(6) Wang, S.-B.; Chen, Z.-X.; Gao, F.; Zhang, C.; Zou, M.-Z.; Ye, J.-J.; Zeng, X.; Zhang, X.-Z., Remodeling Extracellular Matrix Based on Functional Covalent Organic Framework to Enhance Tumor Photodynamic Therapy. Biomaterials 2020, 234, 119772.

(7) Wang, K.; Tu, Y.; Yao, W.; Zong, Q.; Xiao, X.; Yang, R. M.; Jiang, X. Q.; Yuan, Y., Size-Switchable Nanoparticles with Self-Destructive and Tumor Penetration Characteristics for Site-Specific Phototherapy of Cancer. ACS Appl. Mater. Interfaces 2020, 12, 6933-6943.

(8) Chen, M.; Liang, X.; Gao, C.; Zhao, R.; Zhang, N.; Wang, S.; Chen, W.; Zhao, B.; Wang, J.; Dai, Z., Ultrasound Triggered Conversion of Porphyrin/CamptothecinFluoroxyuridine Triad Microbubbles into Nanoparticles Overcomes Multidrug 
Resistance in Colorectal Cancer. ACS Nano 2018, 12, 7312-7326.

(9) Luo, Z.; Tian, H.; Liu, L.; Chen, Z.; Liang, R.; Chen, Z.; Wu, Z.; Ma, A.; Zheng, M.; Cai, L., Tumor-Targeted Hybrid Protein Oxygen Carrier to Simultaneously Enhance Hypoxia-Dampened Chemotherapy and Photodynamic Therapy at a Single Dose. Theranostics 2018, 8, 3584-3596.

(10) Tang, W.; Zhen, Z.; Wang, M.; Wang, H.; Chuang, Y. J.; Zhang, W.; Wang, G. D.; Todd, T.; Cowger, T.; Chen, H.; Liu, L.; Li, Z.; Xie, J., Red Blood Cell-Facilitated Photodynamic Therapy for Cancer Treatment. Adv. Funct. Mater. 2016, 26, 1757-1768. (11) Wang, G. D.; Nguyen, H. T.; Chen, H.; Cox, P. B.; Wang, L.; Nagata, K.; Hao, Z.; Wang, A.; Li, Z.; Xie, J., X-Ray Induced Photodynamic Therapy: A Combination of Radiotherapy and Photodynamic Therapy. Theranostics 2016, 6, 2295-2305.

(12) Zhen, Z.; Tang, W.; Guo, C.; Chen, H.; Lin, X.; Liu, G.; Fei, B.; Chen, X.; Xu, B.; Xie, J., Ferritin Nanocages to Encapsulate and Deliver Photosensitizers for Efficient Photodynamic Therapy against Cancer. ACS Nano 2013, 7, 6988-6996. 